\title{
$\nabla$ \\ IJCRR \\ Anaesthetic Management of a Patient with Multiple Cerebral Hydatid Cyst with Intraventricular Extension: A Case Report
}

Section: Healthcare ISI Impact Factor (2019-20): 1.628

IC Value (2019): 90.81 $\operatorname{SJIF}(2020)=7.893$

(c) (i) (3)

Copyright@IJCRR

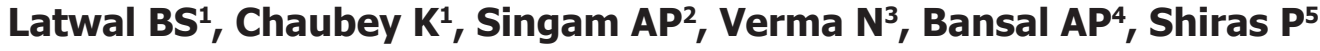

\begin{abstract}
'MD, Senior Resident, Department of Anaesthesiology, JNMC, DMIMS, Wardha, Maharashtra, India; ' MD, PhD, Professor \& Head, Department of Anaesthesiology, JNMC, DMIMS, Wardha, Maharashtra, India; 3DA, MD, Associate Professor, Department of Anaesthesiology, JNMC, DMIMS, Wardha, Maharashtra, India; ${ }^{4}$ MBBS, Junior Resident, Department of Anaesthesiology, JNMC, DMIMS, Wardha, Maharashtra, India; 5] unior Resident, MBBS, Department of Anaesthesiology, JNMC, DMIMS, Wardha, Maharashtra, India.
\end{abstract}

\section{ABSTRACT}

Introduction: One of the worldwide zoonoses affecting humans is Hydatid disease. Echinococcus granulosus, a tapeworm, is the causative organism of Hydatid disease. Humans get this infection either by direct contact with the dog or by eating food that is contaminated with the faeces of the dog. Any organ in the body can be affected by hydatidosis most commonly in the liver and least commonly the brain. Cerebral hydatidosis represents only up to $1 \%$ to $2 \%$ of all hydatid disease cases.

Aims: This case report aims to highlight and focus on the anaesthetic management of a rare case which we do not encounter daily and its management in the preoperative, intraoperative, postoperative period and how to manage complications associated with it.

Case Report: We hereby report a successfully managed case of cerebral hydatidosis which is a rare manifestation of echinococcosis. After inducing the patient with iv anaesthetic agents and intubation, keeping the brain well relaxed, four cerebral hydatid cysts were successfully removed with the avoidance of any intraoperative anaphylactic reactions with all anti anaphylaxis measures.

Discussion: We successfully managed a case of multiloculated multicystic hydatid disease in the left lateral ventricle and adjacent left the periventricular deep white matter in a 20 -year-old female. During the extrusion of third cyst, it was accidentally ruptured which was controlled and managed immediately in an effective manner.A combination of thorough preoperative evaluation, intraoperative monitoring and management to maintain stable haemodynamics and postoperative management and care are essential in such patients for better outcome.

Key Words: Cerebral hydatid cyst, Hydatidosis, Echinococcosis, Hydrodissection, Anaphylaxis

\section{INTRODUCTION}

One of the worldwide zoonoses affecting humans is Hydatid disease (also known as hydatidosis or echinococcosis). Echinococcus granulosus, a tapeworm, is the causative organism of Hydatid disease. Mostly domestic Carnivores, such as dog, are the definite hosts whereas all mammals are intermittent host most commonly sheep and goats. Humans get this infection either by direct contact with the dog or by eating food that is contaminated with faeces of the dog which contains the parasite's ova. It is more prevalent in areas where livestock and dogs are raised together. Any organ in the body can be affected by hydatidosis but the liver is most commonly affected. ${ }^{1,2}$ Other sites are lungs, kidneys, adrenals, pancreas, spleen and brain (least common). ${ }^{2}$ Cerebral hydatidosis represent only up to $1 \%$ to $2 \%$ of all hydatid disease cases and thus is a rare manifestation of echinococcosis. ${ }^{2,3,4}$ The incidence of intracranial hydatid disease in India is $0.2 \%{ }^{5}$ Cyst excision with due care of not to rupture the cyst is the treatment of Hydatid cyst. An alternate option is to aspirate the cystic contents, done in deep-seated cysts and where excision is impossible. ${ }^{1}$

Here we present a case of cerebral hydatidosis in a 20 -yearold female.

\section{CASE REPORT}

A 20-year-old female presented with complaints of severe headache, nausea and giddiness for 1 month. It was occa-

\section{Corresponding Author:}

Chaubey K, MD, Senior Resident, Department of Anaesthesiology, JNMC, DMIMS, Wardha, Maharashtra, India. Email: kashish_chaubey@yahoo.com

ISSN: 2231-2196 (Print) ISSN: 0975-5241 (Online)

Received: $03.11 .2020 \quad$ Revised: 09.01 .2021

Accepted: 20.03 .2021

Published: 20.07 .2021 
sionally associated with non-projectile and non-bilious vomiting. On general examination, the patient was thin built with a weight of $52 \mathrm{~kg}$ and a height of $152 \mathrm{~cm}$. Her pulse rate was $88 / \mathrm{min}$, her blood pressure was $110 / 72$ and she was maintaining saturation of $98 \%$ to $100 \%$ on room air. She was conscious and oriented to time, place and person with a GCS of $15 / 15$. CNS examination showed the power of $4 / 5$ of all four limbs with no sensory deficits. Fundoscopy was done to rule out papilloedema. Her routine blood investigations like complete blood count, kidney and liver function tests showed normal values. Her x-ray of the chest (PA view) and USG abdomen and pelvis were normal and there were no abnormal findings. Liver enzymes levels and PT/INR were also normal like other laboratory reports. MRI contrast of the brain revealed a multiloculated multicystic lesion in the left lateral ventricle and adjacent left the periventricular deep white matter with multiple rounded cystic components [Fig 1]. In the post-contrast study, the lesion shows mild enhancement of septa and wall of the lesion with associated significant mass with midline shift towards the right side and perilesional oedema. There is an associated midline shift of 17 to $18 \mathrm{~mm}$. The neurosurgery team planned for an elective craniotomy and cyst excision.

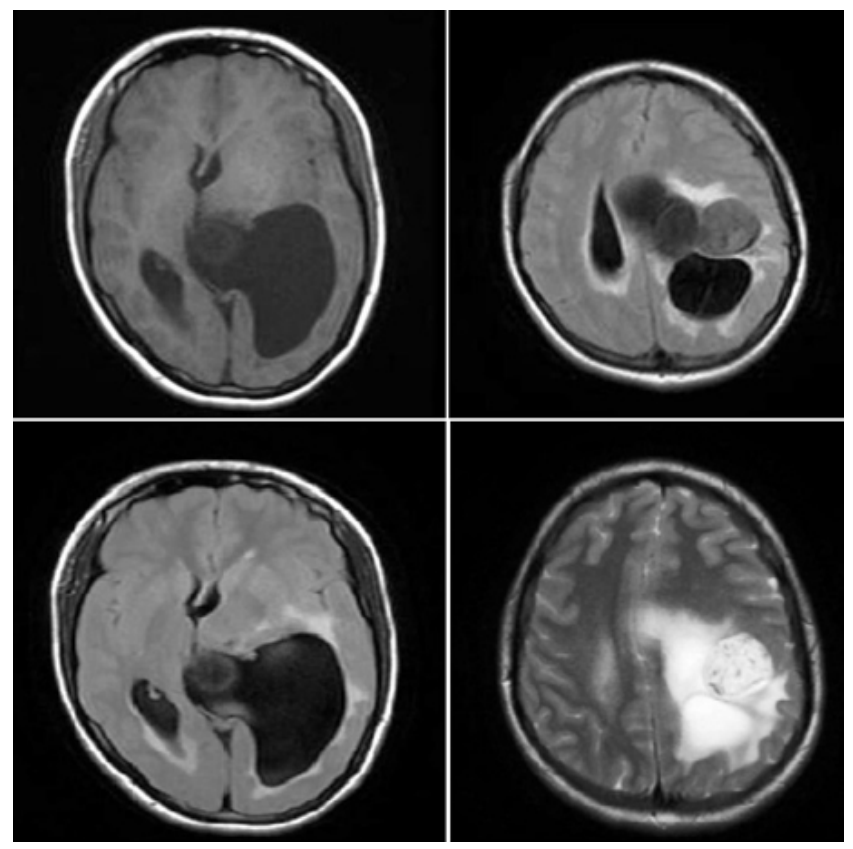

Figure 1: MRI images of the brain (axial view) showing multiloculated multicystic lesion in left lateral ventricle and adjacent left periventricular deep white matter.

\section{ANAESTHETIC MANAGEMENT}

On shifting the patient to the operation theatre, all monitors were attached including arterial BP monitor and reading noted. Two wide bore Intravenous (IV) cannula secured.
Anaphylactic covers were given prophylactically with Inj. Hydrocortisone 100mg IV and Inj. Dexamethasone $8 \mathrm{mg}$ IV. Antianaphylacticmeasures were kept ready which includes adrenaline, antihistaminics and steroids. To treat anaphylactic reactions if presented intraoperatively, drugs like inj. Avil (Pheniramine maleate) and salbutamol (inhaler) were also arranged apart from other emergency medications (epinephrine, atropine and sodium bicarbonate). Antibiotics and antiepileptic were also given before induction. After giving Inj. Glycopyrrolate $0.004 \mathrm{mg} / \mathrm{kg}$ IV, Inj. Midazolam 0.05mg/ $\mathrm{kg}$ IV, Inj. Fentanyl 2microgram/kg IV patient was induced with Inj. Propofol $2 \mathrm{mg} / \mathrm{kg}$ IV and inj. Vecuronium $0.1 \mathrm{mg} / \mathrm{kg}$ IV and sevoflurane of MAC up to $1-1.2$ in a way to keep ETCO2 between 35 to $45 \mathrm{mmHg}$. The patient got intubated with an endotracheal tube of size 7.5. Left temporoparietal craniotomy was performed. Brain wasrelaxed well. Cortisectomy was done along the inferior parietal lobule. At a depth of around $4 \mathrm{~cm}$ anteriorly, a cyst capsule was identified [Fig 2]. Hypertonic saline patties were used to separate the cyst capsule from the brain parenchyma. Hydrodissection was done and two cysts were excised. The posteriorly and inferiorly left ventricle was present. Ventricle decompressed and CSF was released. Ventricle flushed with $3 \% \mathrm{NaCl}$ and hydrocortisone solution. Later superior-anteriorly, evidence of calcified portion with another 2 more cysts was present. While separating the 3rd cyst capsule at the frontoparietal area, the cyst got ruptured. Cyst contents were aspirated with controlled spillage of contents in surrounding brain parenchyma. Both the cyst was excised and the whole surgical field was irrigated with 3\% saline (sodium chloride), betadine and hydrocortisone solution. Haemostasis attained. Dura closed in a watertight fashion. Close monitoring was done during and after the rupture for any signs of anaphylactic reactions. Intraoperative vitals were maintained and remained within normal limits. The patient was haemodynamically stable and there was no increased airway pressure. Hypertonic $\mathrm{NaCl}$ (200 ml) was used as irrigation fluid during the surgery and was continuously suctioned out. The sodium level in the blood was frequently monitored to prevent hypernatremia. There was approximately 400 to $500 \mathrm{ml}$ of blood loss during the surgery and was replaced with 1 unit of PRC and other iv fluids. Postoperatively the patient was reversed with Inj. Myopyrolate $0.05 \mathrm{mg} / \mathrm{kg}$ IV and extubated after fulfilling all extubation criteria and had an uneventful recovery ( Figure 2). The patient was shifted to Neuro ICU for postoperative observation where she was managed with Antibiotics, antiepileptic, analgesic, antacids and other supportive measures. Corticosteroid cover postoperatively continued and tapered off slowly and then stopped. The patient was neurologically and haemodynamically stable and shifted to the ward on the second post-op day. The patient was discharged on postoperative day 6 . Her histopathology report was suggestive of a hydatid cyst (Figure 3 ). 


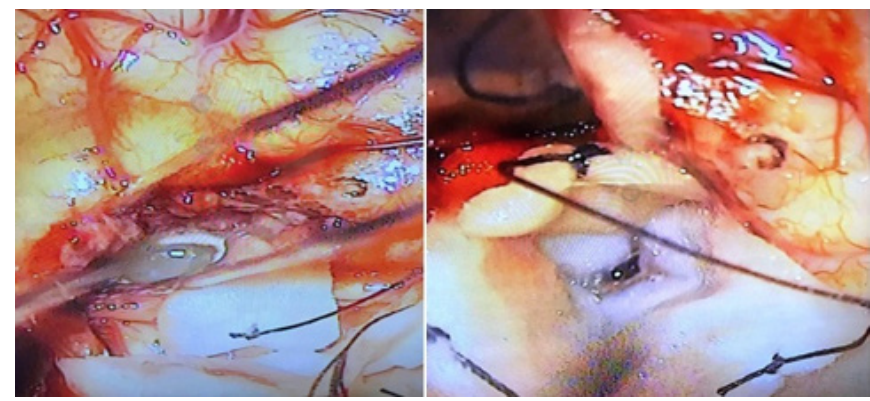

Figure 2: Showing glistening white cyst capsule with hypertonic saline soaked patties.

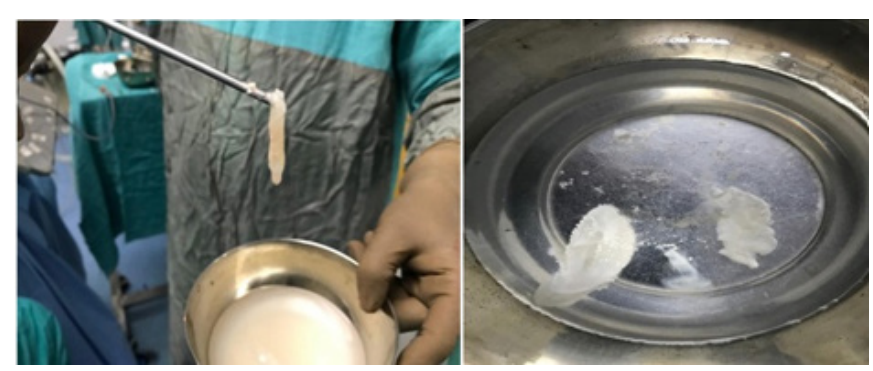

Figure 3: Hydatid cysts after removal.

\section{DISCUSSION}

Cerebral hydatidosis is very rare, with $1-2 \%$ incidence of all hydatid disease cases. ${ }^{4}$ They are mainly located in the parietal lobe, in the supratentorial compartment., ${ }^{2,6}$ Skull, cavernous sinus, eyeball, brainstem, extradural, cerebellum and ventricles are the other less common sites. ${ }^{2,3,5}$ Cerebral involvement occurs mainly in the pediatric age groups and is rare in adults. ${ }^{5}$ Solitary intracranial cysts are common than multiple intracranial cysts. Cerebral hydatid cysts are classified as primary (single) or secondary (multiple). ${ }^{6,7}, 8$ Primary cerebral hydatid cysts are formed because of the direct larval infestation in the brain parenchyma without any other organ involvement. Primary cysts contain scolices, which are fertile. Therefore rupture of a primary cyst can cause dissemination and recurrence. Multiple cerebral hydatid cysts are rare and are usually secondary resulting from the rupture of a solitary primary cerebral hydatid cyst or can result from embolization of cyst in some other organ. These secondary cysts are infertile and lack live scolices. ${ }^{3,6,7}$ Primary multiple hydatid cysts are extremely rare. The patients usually present with headache, nausea and vomiting (due to raised Intracranial tension), neurological deficits (due to local compressive effects), hemiparesis, visual disturbances like double vision and seizures according to cyst's size, number, location and host immune response to the cyst. ${ }^{3,9}$

Surgery aims at the intact delivery of cerebral hydatid cysts in all cases. Many surgical techniques are used for removing the hydatid cyst. The most popular and recommended is Dowling's hydro dissection technique where irrigation with normal saline is used to deliver the cyst. ${ }^{3,6,7,8,10}$ Another method is direct puncture with aspirating the cystic contents via a small hole created in the cyst wall and removal of the cyst. ${ }^{6}$ In our case, hydro dissection was done by $3 \%$ hypertonic saline, which by keeping the cyst in a hyperosmolar environment decreases the tension inside the cyst. The incision and retraction of the cerebral cortex to reach the cyst wall may lead to (transient or permanent) neurological deficits. ${ }^{2}$ Hydrodissection of the cyst gently by irrigating fluid between cyst wall and brain interface is suggested if the cyst is separated from the brain. ${ }^{6,8,10}$ In our case, the surgeon used Dowling's hydro dissection technique for the intact removal of hydatid cysts.

Aims of anaesthesia are to keep the brain in a relaxed state intraoperatively and to hinder the patient movement during the procedure and to provide a surgical field that is devoid of bleeding thus assisting the surgeon to excise the cyst smoothly. ${ }^{6}$ Some of the anaesthetic challenges which may occur during manipulation and resection of hydatid cyst include hemodynamic instability, anaphylaxis due to the rupture of the cyst and massive bleeding due to the relieving of pressure from the vessels after the excision of the cyst. ${ }^{7-10}$

To prevent or avoid the recurrence of hydatid cysts due to the rupture and release of protoscolices of the parasite various sporicidal agents can be used. One such sporicidal agent is hypertonic saline which is very effective but can cause hypernatremia which may lead to seizures and dehydration of brain cells. ${ }^{11}$ Hence frequent intraoperative and postoperative serum sodium monitoring is required. To prevent these complications, a lesser concentration of hypertonic saline (3\%) was used and monitored serum sodium level at regular intervals. Hydrogen peroxide $\left(\mathrm{H}_{2} \mathrm{O}_{2}\right)$ is also an acaricidal agent. ${ }^{12}$ Oxygen released from $\mathrm{H}_{2} \mathrm{O}_{2}$ exhibits sporicidal action. In some studies, it was found that hydrogen peroxide can cause gaseous embolism and hemodynamic instability. ${ }^{12}$

Some of the complications which can occur perioperatively are rupture of the hydatid cyst, allergic or anaphylactic reaction, subdural effusion, subdural hematoma, epidural hematoma, bacterial infection, seizures and pneumocephalus. ${ }^{1,4,6}$ Allergic reaction, ranging from mild hypersensitivity to life-threatening anaphylaxis, is the major complication that occurs due to the accidental rupture of hydatid cyst intraoperatively. ${ }^{1}$ Rupture results in the release of highly antigenic contents of the hydatid cyst into the bloodstream leading to IgE mediated anaphylactic reactions which cause hypotension (severe) and bronchospasm (increasing airway pressure). ${ }^{1,6,8}$ In case of accidental rupture of hydatid cyst, the cyst contents should be suctioned out carefully and irrigate the entire operative field with $3 \%$ hypertonic $\mathrm{NaCl}^{3,7}$ Definite treatments for life-threatening anaphylaxis include - administration of intravenous epinephrine with $100 \%$ oxygen administration and Hydrocortisone and massive fluid resus- 
citation. ${ }^{1,6}$ Medical treatment with albendazole is beneficial both pre and postoperatively. ${ }^{1,2,3}$

\section{CONCLUSION}

Here, we successfully managed a case of multiloculated multicystic hydatid disease in the left lateral ventricle and adjacent left the periventricular deep white matter in a 20 -yearold female with complaints of headache, giddiness, nausea and vomiting. During the extrusion of the third cyst, it was accidentally ruptured which was controlled and managed immediately in an effective manner. Conclusively, a combination of thorough preoperative evaluation, intraoperative monitoring and management to maintain stable haemodynamics and postoperative management and care are essential in such patients for better outcome.

\section{ACKNOWLEDGEMENT}

Authors acknowledge the immense help received from the scholars whose articles are cited and included in references of this manuscript. The authors are also grateful to authors / editors / publishers of all those articles, journals and books from where the literature for this article has been reviewed and discussed.

\section{Conflict of Interest: None}

\section{Source of Funding: Nil}

Authors Contribution:

Basant Singh Latwal - Primary Author, Data Collection and Analysis, Compilation, Case Management in the OT

Kashish Chaubey - Corresponding Author, Data Collection and Analysis, Compilation, Case Management in the OT

Amol P Singam - Data Interpretation, Head of the Team Supervising the Case, Final Review of the Case Report to be Published

Neeta Verma - Data Analysis and Interpretation, Conception of or Design of the Case Report
Ayush Pal Bansal - Data Collection, Analysis and Interpretation

Shiras P-Collection, Analysis and Interpretation

\section{REFERENCES}

1. Nashibi M, Tafrishinejad A, Hussain Khan Z. Deep Seated Cerebral Hydatid Cyst and Its Anesthetic Considerations: A Case Report. Archiv Neurosci.. 2020 Jan 31;7(1).

2. Dr. Babita, Dr. Shobhapurohit, Dr. Bhupendrasingh. Anaesthetic Management of intracranial Hydrated Cyst, A Patient of Disseminated Hydatid Disease ${ }^{-}$A Case Report. Ind J Appl Res. 2014;4(5).

3. Kandemirli SG, Cingoz M, Olmaz B, Akdogan E, Cengiz M. Cerebral Hydatid Cyst with Intraventricular Extension: A Case Report. J Trop Pediat. 2019;65(5):514-519.

4. Tuzun Y, Kadioglu HH, Izci Y, Suma S, Keles M, Aydin IH. The clinical, radiological and surgical aspects of cerebral hydatid cysts in children. Pediat Neuros. 2004;40(4):155-160.

5. Pandey S, Pandey D, Shende N, Sahu A, Sharma V. Cerebral intraventricular echinococcosis in an adult. Surg Neurol Intern. 2015;6.

6. Panda NB, Batra YK, Mishra A, Dhandapani SS. A giant intracranial hydatid cyst in a child: Intraoperative anaesthetic concerns. Ind J Ana. 2014;58(4):477.

7. Yurt A, Avcı M, Selçuki M, Özer F, Çamlar M, Uçar K, et al. Multiple cerebral hydatid cysts: report of a case with 24 pieces. Clin Neur Neuros. 2007;109(9):821-826.

8. Gupta S, Desai K, Goel A. Intracranial hydatid cyst: a report of five cases and review of the literature. Neur Ind. 1999;47(3):214.

9. Padayachy LC, Dattatraya M. Hydatid disease (Echinococcus) of the central nervous system. Child's Nerv Syst. 2018;34(10):1967-1971.

10. Carrea R, Dowling jr E, Guevara JA. Surgical treatment of hydatid cysts of the central nervous system in the pediatric age (Dowling's technique). Ped Neuros. 1975;1(1):4-21.

11. Albi A, Baudin F, Matmar M, Archambeau D, Ozier Y. Severe hypernatremia after hypertonic saline irrigation of hydatid cysts. Anesth Analg. 2002;95(6):1806-1808.

12. Ouerghi S, Abdelhafidh K, Merghli A, Smati B, Boudaya MS, Lamine $\mathrm{K}$, et al. Iatrogenic gas embolism after use of hydrogen peroxide in the treatment of lung hydatid cyst: a report of 2 cases. La Tunisie Med. 2010;88(11):851-854. 